\title{
Arbor
}

\section{Lo público y lo privado en Sanidad}

\section{Carlos Pérez Espuelas}

Arbor CLXX, 670 (Octubre 2001), 353-362 pp.

La experiencia desarrollada en ambos campos, permite al autor de este artículo dar una visión positiva de la aportación que el sector privado puede realizar de forma creciente a los objetivos del sistema sanitario, estableciendo factores de competitividad, con una más eficiente utilización de recursos, apostando por la apertura hacia nuevas formas de provisión, en un marco de libertad de elección.

\section{Presentación}

Nuestro objetivo con este breve artículo es analizar el posicionamiento de los sectores público y privado en el aseguramiento y provisión de servicios sanitarios, junto con su evolución en los últimos años, así como proporcionar una opinión sobre las tendencias de futuro. Pretendemos analizar cómo el sector privado y público se han relacionado, el papel que a cada uno de ellos le ha reservado el Estado en este tiempo, y trataremos de mostrar de qué forma el sector privado ha trabajado, y trabaja, codo a codo con el sector público, proporcionando a los ciudadanos aseguramiento y/o atención sanitaria de calidad, complementándose, y aportando el sector privado referentes de estándares tanto de atención al cliente como de gestión de recursos. 
La separación entre aseguramiento privado y público no es nítida para toda la población, como es el caso del colectivo de personal funcionario que tiene opción para recibir tanto el aseguramiento como la atención sanitaria a través de empresas de seguro privado. De la misma forma, los colectivos de una serie de empresas (que reciben el nombre de Colaboradoras) obtienen a través del seguro privado la atención médica. Estas excepciones están siendo motivo de un intenso debate político, constituyéndose en el núcleo de la discusión Sanidad Privada versus Sanidad Pública, dado que ya en este momento la atención sanitaria de los españoles se financia desde los presupuestos generales del Estado, dando lugar al argumento de que no deben existir diferencias entre las personas. Entendemos que si el análisis se efectuara alrededor de la libertad de elección de los ciudadanos estos optarían, como tantas veces han demostrado, por la liberación del sector y la competitividad, lo que se traduciría en un mejor servicio, que es finalmente lo que se espera recibir.

Mayor confusión encontramos en la provisión, hasta tal punto que aquí lo público y lo privado se entremezcla con lo privado no lucrativo y lo público lucrativo (empresas públicas, consorcios, etc), si bien este lucro tiene una forma de reparto distinta del privado, finalmente determina que asistamos a una sana competencia por la provisión, en la que, como en el caso de Cataluña, están obligados a participar todos los sectores. Hemos asistido a un crecimiento complementario de los proveedores, supliendo la iniciativa privada carencias del sector público en aspectos esenciales tanto del diagnóstico como del tratamiento, enmarcado siempre en el discurso político privado/público (que tantas veces enmascara la presión por el mantenimiento de la condición de empleado público), lo que ha dificultado una colaboración a largo plazo entre ambos sectores., ya que no siempre han prevalecido los criterios de planificación estratégica o económicos.

\section{Introducción}

El sector privado participa de distintas formas en la asistencia sanitaria; así, en el aseguramiento proporciona cobertura sanitaria a los ciudadanos, actuando por tanto en condiciones de doble asegurador; en la provisión, prestando atención sanitaria a ciudadanos con aseguramiento público (MUFACE, ISFAS, MUGEJU) y privado; y más recientemente, financiando la provisión pública (caso de Alcira en Valencia). Y estas tareas las realiza a través de empresas privadas con y sin ánimo de lucro. 
El sector público asegurador, proporciona la atención sanitaria a través de centros propios; también mediante empresas públicas, consorcios, fundaciones, etc, donde la propiedad es de Entes Locales, Provinciales, Cabildos.... Estas últimas se diferencian de las empresas privadas sin ánimo de lucro en las relaciones laborales (funcionariales en muchos casos), la carencia de personalidad jurídica propia de alguna de ellas, etc.

Pero el sector público también proporciona atención a pacientes privados, bien al ser remitidos por sus compañías de seguro médico o por compañías de seguro de accidentes, de accidentes de tráfico, etc. Finalmente, el Estado, como asegurador público, proporciona asistencia sanitaria a través de hospitales y servicios privados concertados.

Desde la óptica de la gestión, al tratarse de una actividad mayoritariamente pública, se basa en principios de gestión pública. Este hecho objetivo condiciona e influye de manera muy significativa las formas en las que se producen los esquemas de gestión sanitaria. No se trata de juzgar aquí si esta circunstancia es globalmente positiva, negativa, adecuada, etc; simplemente marca una serie de elementos clave de referencia, entre los que cabría señalar:

- Las dificultades objetivas para orientar los focos de atención hacia el paciente/cliente

- La pérdida de referencia del mercado como elemento de ajuste

- La menor eficacia e importancia del uso de indicadores económicofinancieros como elementos de medición.

- El menor impacto de los mecanismos de la competencia como elemento dinamizador.

- Las características específicas del marco de gestión de recursos humanos en las organizaciones sometidas a los criterios de gestión pública.

\section{Antecedentes históricos}

Para analizar la evolución de la atención sanitaria en España en estos últimos 20 años debe comenzarse por nuestra Carta Magna, en cuyo artículo 43 reconoce el derecho de los españoles a la protección de la salud, estableciendo la competencia de los diferentes niveles de los poderes públicos para organizar, y hacer cumplir este derecho. La organización y tutela, por parte de las Administraciones Públicas, de la salud pública se realiza a través de medidas preventivas y de las prestaciones y servicios necesarios. La Constitución establece que el Estado es asegurador sanitario de todos los españoles. 
La generalización de la cobertura sanitaria pública tiene su concreción legal en la Ley General de Sanidad de 24 de abril de 1986. Hasta la promulgación de la misma, el servicio público de salud estaba ligado al sistema contributivo de la Seguridad Social, y por tanto al mundo del trabajo, a través del Seguro Obligatorio de Enfermedad creado en el año 1942. La afiliación al sistema era requisito básico para poder disfrutar de dicho servicio público.

\section{La financiación}

En la década de los 60 el porcentaje de gasto sanitario público situó en el 59\%, y en los 70 en el $65 \%$ del total. A partir de la década de los 80 , este porcentaje se elevó al $80 \%$, mostrando un descenso del $5 \%$ a partir del año 95 (OCDE Health Data). La aportación del Estado a la financiación del gasto sanitario público cuando se suscribieron los Pactos de la Moncloa en 1977 era del 20\%, del gasto, y el 80\% restante provenía de las aportaciones del Sistema de Seguridad Social.

Esta participación mantiene una tendencia progresiva, y a partir del año 90 pasa a tener un carácter finalista, de tal forma que se financia parte del gasto sanitario con independencia de otras aportaciones destinadas a financiar prestaciones económicas de carácter no contributivo, separando la financiación de la Sanidad de la de Seguridad Social.

La participación de las cotizaciones sociales va perdiendo peso a lo largo de la década de los 90 hasta desaparecer en 1999, cumpliéndose de esta forma lo establecido en el Pacto de Toledo. En consecuencia, desde el año pasado el gasto sanitario público en España se financia en su totalidad desde los presupuestos generales del Estado.

\section{Gasto sanitario en nuestro entorno}

Lluis Bohigas analiza la evolución del gasto sanitario de los años 95 y 96 en España, y los compara con la media europea, donde encuentra valores muy próximos. También analiza la diferencia entre gasto sanitario público y privado para el mismo periodo, como se recoge en el siguiente cuadro.

Bohigas concluye que el esfuerzo que realiza España en cuanto a PIB dedicado a la sanidad es equivalente a la media de los países de la Unión Europea, estando más cerca de la media europea en gasto privado que en gasto público. 
Lo público y lo privado en Sanidad

\begin{tabular}{|l|c|c|}
\hline & Año 95 & Año 96 \\
\hline Gasto Sanitario en España en \% del PIB & 7,6 & 7,7 \\
\hline Gasto Sanitario en Europa en \% del PIB & 7,8 & 7,9 \\
\hline Gasto sanitario público en España en \% del PIB & 6,0 & 5,9 \\
\hline Gasto sanitario público en Europa en \% del PIB & 6,1 & 6,1 \\
\hline Gasto sanitario privado en España en \% del PIB & 1,6 & 1,8 \\
\hline Gasto sanitario privado en Europa en \% del PIB & 1,8 & 1,8 \\
\hline
\end{tabular}

De esta información podemos concluir que nos encontramos en un sistema sanitario donde el peso del sector público es mayoritario, en el que todos los ciudadanos tienen garantizada la asistencia sanitaria, que convive con un sector privado minoritario, y que es equiparable a la media europea, en especial en gasto privado.

\section{Los recursos y la actividad en provisión}

El total de hospitales que conforman el censo nacional es de 799, de los cuales, el $18 \%$ son privados benéficos y el $34 \%$ son privados no benéficos. Este porcentaje se modifica si consideramos el número de camas, y de un total de 166.276, privadas benéficas son el $13 \%$ y privadas no benéficas el $17 \%$ (Censo de hospitales del Ministerio de Sanidad y Consumo 1999). De nuevo observamos el elevado peso del sector no público en provisión, con su indudable repercusión en la economía nacional.

En el cuadro siguiente recogemos las altas hospitalarias del año 96 con arreglo al régimen de financiación, y clasificadas según la dependencia del hospital. Se aprecia como se distribuye el mapa de la actividad hospitalaria, y puede observarse el grado de concentración entre aseguradoras y proveedores, de tal forma que, mayoritariamente, cada sector utiliza sus propios recursos, sin que por ello se pueda considerar no significativa la interalación, puesto que el $13 \%$ de la actividad de Seguridad Social efectuada por privados es muy importante.

Para el INSALUD no transferido, los gastos en Asistencia Sanitaria con Medios Ajenos se han estabilizado en el $10 \%$ del presupuesto, y viene a representar en el último ejercicio, unos 140.000 millones de pesetas. 
Carlos Pérez Espuelas

\begin{tabular}{|l|c|c|c|c|c|}
\hline & $\begin{array}{c}\text { Pacientes } \\
\text { propios }\end{array}$ & $\begin{array}{c}\text { Aseguradoras } \\
\text { privadas }\end{array}$ & $\begin{array}{c}\text { Seguridad } \\
\text { social }\end{array}$ & $\begin{array}{c}\text { Mutuas de } \\
\text { funcionarios }\end{array}$ & $\begin{array}{c}\text { Entidades } \\
\text { públicas }\end{array}$ \\
\hline Sistema Nacional de Salud & $10 \%$ & $2 \%$ & $66 \%$ & $10 \%$ & $11 \%$ \\
\hline Otros públicos & $3 \%$ & $1 \%$ & $21 \%$ & $77 \%$ & $73 \%$ \\
\hline Privados benéficos & $19 \%$ & $17 \%$ & $7 \%$ & $2 \%$ & $3 \%$ \\
\hline Privados no benéficos & $68 \%$ & $81 \%$ & $6 \%$ & $11 \%$ & $13 \%$ \\
\hline
\end{tabular}

Fuente: Ministerio de Sanidad y Consumo.

\section{La concertación de medios ajenos al Estado}

El Estado ha venido supliendo su falta de medios para prestar la atención sanitaria mediante la concertación con servicios ajenos. Así, en la Ley General de Seguridad Social, Texto Refundido de 30 de mayo de 1974, establece en su artículo 104 que «la asistencia en régimen de internado se hará efectiva en las Instituciones Sanitarias de la Seguridad Social o mediante concierto», y este se podrá establecer tanto con entidades públicas como privadas. Pero no se desarrolla más el articulado, por lo que en abril de 1980 se publica la Resolución de la Secretaría de Estado para la Sanidad, y con ella se regula la prestación de asistencia sanitaria en Centros ajenos, y se determina el carácter subsidiario y complementario de la misma, otorgando prioridad para ello a los centros del sector público y a los del privado sin ánimo de lucro.

La Resolución de la Secretaría de Estado significa el primer intento de racionalizar las relaciones con el sector privado, clasificando a los centros en grupos y niveles, estableciendo las tarifas en función de dicha clasificación, y definiendo el papel que las instituciones privadas desempeñarán en el futuro dentro de la provisión de servicios al Estado.

La Ley General de Sanidad supone un nuevo avance en la relación entre sector privado y público. Así, reconoce la posibilidad de que los hospitales generales privados puedan vincularse al sector público conservando la titularidad de los Centros y relaciones laborales. Establece en su artículo 67 que la vinculación se realizará mediante un convenio singular. Y a partir de la Orden de Revisión de Tarifas de 1993 se introduce la concertación de una serie de procedimientos quirúrgicos concretos con sus correspondientes tarifas. Más recientemente el INSALUD ha enmarcado los conciertos dentro de los contratos de gestión de servicios públicos acogiéndose a lo establecido dentro de la Ley de Contratos de las Administraciones Públicas. 


\section{La experiencia catalana en provisión}

En el año 1981 se produce la transferencia sanitaria a Cataluña, pasando con ello a gestionar tanto los recursos como el presupuesto. En Cataluña en el momento de la transferencia, solo un $30 \%$ de los hospitales eran del Estado, y el resto eran de otras organizaciones públicas, y privados sin y con ánimo de lucro. Esta realidad determinó que en el año 1985 la Generalitat crease la red hospitalaria de utilización pública, y separase las funciones de provisión de las de compra, y aplica los mismos criterios de contratación de servicios a su red propia (Instituto Catalán de la Salud) que compite en igual de condiciones con el resto de hospitales. El Servicio Catalán de la Salud es el encargado de gestionar la compra de servicios.

En el año 97, el presupuesto del servicio de salud supuso el $34,5 \%$ del total del presupuesto de la Generalitat, lo que nos proporciona una clara idea de la importancia y peso del sector en el conjunto competencial del gobierno catalán.

\section{La experiencia valenciana en provisión}

Hemos visto como tanto la Administración Central como la Autonómica, han resuelto la cobertura asistencial con los medios que disponían, de tal forma que han utilizado los recursos disponibles para adecuarse a la demanda, pero cuando han tenido la necesidad de crecer, lo han hecho construyendo hospitales de propiedad pública, y gestión pública.

Es en la Comunidad Autónoma Valenciana donde se ha dado un paso adelante en la liberación de la provisión, y para proporcionar asistencia sanitaria a una población de 230.000 habitantes en la Comarca de la Ribera (Valencia), se crea una Unión Temporal de Empresas, liderada por una compañía de seguros privada. El modelo se basa en una financiación pública (pago capitativo), control público, propiedad pública (construido sobre un solar público, revertirá a la Generalitat en el plazo de 10 años), y la prestación del servicio es privada, por tanto, una gestión empresarial, con relaciones laborales.

Esta forma de colaboración del sector privado con el público, mediante la formación de alianzas estratégicas a largo plazo, supone una apuesta interesante y novedosa, que debería extenderse al resto de Comunidades, permitiendo la renovación y mejora de la gestión del sector hospitalario en su conjunto. 


\section{Carlos Pérez Espuelas}

\section{El aseguramiento privado}

Según UNESPA (Información Estadística del Seguro Privado 1997), el número de pólizas de seguros de enfermedad ha pasado de 2,4 a 4 millones entre los años 1988 y 1997, siendo el número de asegurados para el último año de 9,2 millones de personas. Podemos observar como en los últimos 10 años el sector ha experimentado un crecimiento en el número de pólizas del $70 \%$, asegurando el $20 \%$ de la población española.

Esta actividad supone que el sector recaude un total de 322.000 millones en primas. Con una siniestralidad sobre primas del $82,4 \%$, y unos gastos de gestión del $19 \%$ en el año 97 , el sector tiene un escaso margen de beneficio, muy por debajo de otros sectores industriales y de servicio.

\section{Propuestas presentadas}

Finalmente, analizamos las propuestas más significativas que podemos encontrar acerca de la relación entre sectores público y privado en Sanidad.

La política SALUD21 para la Región Europea de la OMS selecciona cuatro principales estrategias de acción, y una de ellas establece «atención primaria de salud orientada a la comunidad y a la familia con un carácter integrado, con la asistencia de un sistema hospitalario flexible y con capacidad de respuesta». Y en el «objetivo $17^{\circ}$. financiar servicios sanitarios y asignar recursos» de SALUD21, la OMS hace propios los acuerdos de la Conferencia de Ljubljana de 1996 sobre la reforma de la atención sanitaria, al afirmar que «no hay espacio para una actividad sin restricciones del mercado ni en la financiación ni en la provisión de un bien social como la atención sanitaria. Además, los mecanismos de mercado dirigidos a las personas o a los organismos de financiación han tenido mucho menos éxito en términos de equidad $\mathrm{y}$ eficiencia que los dirigidos a hospitales y a otros proveedores de atención sanitaria».

Michael E. Porter, en su libro Ser Competitivo (ediciones Deusto) afirma que «la competencia únicamente funcionará cuando las decisiones tomadas por proveedores, médicos, pagadores y pacientes se basen en información pertinente y comparable sobre precios y sobre resultados terapéuticos».

Andersen Consulting en colaboración con Burson-Marsteller publicaban el 1993 un informe bajo el título «El Futuro de la Sanidad en 
Europa», donde identifican que en las reformas introducidas recientemente en Europa se pretende «promover la competencia entre los dispensadores públicos como medida para incrementar su flexibilidad y eficiencia». Y observan que «adicionalmente, en algunos países se podría alentar la competencia entre los aseguradores públicos y privados, y aumentar la libertad de elección en el tipo de cobertura».

También la consultora Coopers\&Lybrand en 1995 publica «European healthcare trends: towards managed care in Europe», y concluye que «la implantación de competencia requiere individuos bien informados y agencias capaces de efectuar la selección coste-beneficio para la comunidad. Tanto los individuos como las agencias deben ser capaces de influir sobre los resultados de los proveedores de salud».

Un equipo de profesores de ESADE bajo la coordinación de Rafael Bengoa, publicaron en 1997 «Sanidad, la reforma posible», y en el que proponían «abrir un debate constructivo sobre el papel de la medicina privada. Una revisión del rol y las normas que regulen la cooperación del sector no público pueden aliviar el gasto y favorecer niveles de competencia».

La sociedad civil española también se ha pronunciado sobre su consideración en la relación entre sector público y privado. En el año 1991, la Comisión de Análisis y Evaluación del Sistema Nacional de Salud creada por el Parlamento, más conocida como «Informe Abril» establece que «la provisión de asistencia sanitaria al S.N.S. con medios distintos de los públicos, y desarrollada hasta la fecha en compartimentos muy estancos, tiene que ser el soporte de la complementariedad y un elemento necesario para devolver eficiencia al sistema en general, debiendo entrar aún régimen de mayor participación y competencia leal».

En diciembre de 1997, se produjo el acuerdo parlamentario para la Consolidación y Modernización del Sistema Nacional de Salud, donde se acordó recomendar al Gobierno «impulsar la competencia entre proveedores en el marco de un mercado sanitario regulado, con el soporte de un sistema integrado de información, fomentando relaciones estables y duraderas entre los agentes del sistema, instrumentando a través del control y evaluación de los resultados obtenidos».

\section{Conclusiones}

Podemos afirmar que el sector privado y el público han mantenido una continuada colaboración, rellenando aquél los nichos de atención de éste, compartiendo profesionales y experiencias. La paulatina incorporación de reglas de mercado en el sector público derivado de la 


\section{Carlos Pérez Espuelas}

necesidad de mejorar la eficiencia del mismo, exigirá la inclusión de elementos de competencia regulada, la implantación de relaciones laborales adecuadas a la realidad social de nuestros días, etc., todo ello ayudará a incrementar el papel del sector privado, como consecuencia de la paulatina apertura de la provisión. La incipiente incorporación del sector privado a la provisión en atención primaria ayudará a la liberación de la provisión también a este nivel asistencial.

La progresiva demanda de libertad de elección de los ciudadanos será otro elemento que empujará a la apertura del sector público, permitiendo que se ponga de manifiesto como la mayor eficiencia de los hospitales privados es un activo valorado positivamente por los pacientes. A su vez, supondrá el acicate para la reforma del sector público.

También las necesidades de financiación del sector público ayudará en la progresiva incorporación del sector privado tanto en el aseguramiento como en la provisión. En el año 1999 se ha podido apreciar cómo al modificar la fiscalidad sobre el seguro privado, se ha producido un importante incremento de pólizas, lo que se traducirá en un menor consumo de recursos públicos, y tal vez sea esta la vía por la que se inicie la solución al problema de la financiación. Existe una demanda latente de atención privada, y una mínima incentivación fiscal a la misma lo ha puesto de manifiesto. De la misma forma como la experiencia de Alcira se está consolidando, el sector privado tendrá interés en incrementar su presencia en la provisión directa, y habremos de asistir a otras muestras similares de colaboración a largo plazo, conformándose así alianzas estratégicas donde la confianza mutua es elemento imprescindible para su materialización. 